\title{
Biodegradable rifampicin-releasing coating of surgical meshes for the prevention of bacterial infections
}

This article was published in the following Dove Press journal:

Drug Design, Development and Therapy

18 September 2017

Number of times this article has been viewed

\author{
Jochen Reinbold' \\ Teresa Hierlemann' \\ Lukas Urich' \\ Ann-Kristin Uhde' \\ Ingrid Müller ${ }^{2}$ \\ Tobias Weindl ${ }^{3}$ \\ Ulrich Vogel ${ }^{4}$ \\ Christian Schlensak' \\ Hans Peter Wendel' \\ Stefanie Krajewski' \\ 'Department of Thoracic and \\ Cardiovascular Surgery, University \\ Hospital Tübingen, Tübingen, \\ ${ }^{2}$ Department of Pharmaceutical \\ Engineering, Albstadt-Sigmaringen \\ University of Applied Science, \\ Albstadt, ${ }^{3}$ Aimecs ${ }^{\circledR} \mathrm{GmbH}$ Medical \\ Solutions, Pfarrkirchen, ${ }^{4}$ Institute \\ of Pathology and Neuropathology, \\ Tübingen, Germany
}

\begin{abstract}
Polypropylene mesh implants are routinely used to repair abdominal wall defects or incisional hernia. However, complications associated with mesh implantation, such as meshrelated infections, can cause serious problems and may require complete surgical removal. Hence, the aim of the present study was the development of a safe and efficient coating to reduce postoperative mesh infections. Biodegradable poly(lactide-co-glycolide acid) microspheres loaded with rifampicin as an antibacterial agent were prepared through single emulsion evaporation method. The particle size distribution $(67.93 \pm 3.39 \mu \mathrm{m}$ for rifampicin-loaded microspheres and $64.43 \pm 3.61 \mu \mathrm{m}$ for unloaded microspheres) was measured by laser diffraction. Furthermore, the encapsulation efficiency of rifampicin $(61.5 \% \pm 2.58 \%)$ was detected via ultraviolet-visible (UV/Vis) spectroscopy. The drug release of rifampicin-loaded microspheres was detected by $\mathrm{UV} / \mathrm{V}$ is spectroscopy over a period of 60 days. After 60 days, $92.40 \% \pm 3.54 \%$ of the encapsulated rifampicin has been continuously released. The viability of BJ fibroblasts after incubation with unloaded and rifampicin-loaded microspheres was investigated using an MTT (3-(4,5dimethylthiazol-2-yl)-2,5-diphenyltetrazolium bromide) assay, which showed no adverse effects on the cells. Furthermore, the antibacterial impact of rifampicin-loaded microspheres and mesh implants, coated with the antibacterial microspheres, was investigated using an agar diffusion model with Staphylococcus aureus. The coated mesh implants were also tested in an in vivo mouse model of staphylococcal infection and resulted in a $100 \%$ protection against mesh implant infections or biofilm formation shown by macroscopic imaging, scanning electron microscopy, and histological examinations. This effective antibacterial mesh coating combining the benefit of a controlled drug delivery system and a potent antibacterial agent possesses the ability to significantly reduce postoperative implant infections.
\end{abstract}

Keywords: postoperative infections, drug delivery system, antibacterial implant coating, Staphylococcus aureus, antibiotic

\section{Introduction}

In the last decade, polypropylene (PP) mesh implants have been extensively used as prosthetic biomaterials to support and repair abdominal wall defects, such as hernias. These types of surgical operations to reinforce the soft tissue are common procedures in industrialized countries, and they are performed more than one million times per year worldwide. ${ }^{1}$ One important factor affecting the success rate of surgical operations is the appearance of a postoperative wound infection, ${ }^{2,3}$ which occurs in up to $9 \%$ of cases. ${ }^{4}$ Following a mesh-related infection, regular antibiotics are administered systemically. If this therapy fails, a surgical removal of the infected mesh implant is necessary to protect the patient from serious complications. Even today, after years of

\footnotetext{
Correspondence: Stefanie Krajewsk Department of Thoracic and Cardiovascular Surgery, University Hospital Tübingen, Calwerstr 7/I, 72076 Tübingen, Germany

$\mathrm{Tel}+49707 \mathrm{I} 2983303$

Fax +49 707I 293617

Email stefanie.krajewski@

uni-tuebingen.de
} 
using systemic antibiotics to reduce mesh-related infections, their application is still debated, ${ }^{5}$ as the intensive use of broad-spectrum antibiotics leads to a faster and more frequent formation of antibiotic-resistant bacteria strains.

Alternatively, the local use of prophylactic antibiotics applied to the implant surface may reduce the risk of postoperative mesh infections and decrease the formation of antibiotic-resistant bacteria strains. Besides, a local drug delivery system is of huge benefit, as systemic antibiotic administration to treat postoperative infections may be ineffective in patients with poorly vascularized infected tissues. Furthermore, normal doses could be insufficient against biofilms on implants or afflicted tissues. ${ }^{6,7}$ Using a local drug delivery approach, antibiotics are entrapped in a drug delivery system in the form of nanoparticles, microspheres, or coated implants.

Poly(lactide-co-glycolide acid) (PLGA), a biodegradable polymer that has been applied for drug delivery, is mainly used due to its good biodegradability, biocompatibility, and toxicological properties in medicine and pharmacy. Furthermore, the amount of active ingredients encapsulated, their release, and thus the biological distribution can be well controlled. ${ }^{8-10}$ PLGA is also one of a few polymers approved by the US Food and Drug Administration for clinical use in humans. Microspheres, nanoparticles, or implant coatings can be produced using PLGA and applied as a drug delivery system exhibiting controlled release of the encapsulated drug over a few days to months for local application. ${ }^{11,12}$ Such an application reduces the amount of antibiotic in the body, while increasing the concentration in the affected area, whereby the negative side effects are reduced. ${ }^{7}$ For this, the development of a novel drug delivery system needs to be implemented with highly efficient antibiotics available today. ${ }^{13}$ One method to prepare microspheres as a local drug delivery system is the emulsion evaporation method. ${ }^{14}$ This preparation method allows the microspheres to emerge from a single emulsion with an organic and an aqueous phase during the vaporization of the organic solvent. ${ }^{15}$

Rifampicin, an antibiotic agent first synthesized in 1965, has excellent antibacterial properties against Mycobacterium tuberculosis and a broad antibacterial spectrum against Gram-positive and Gram-negative bacteria. It is a liposoluble antibiotic, thus penetrating most tissues. The antibacterial mechanism of action relies on the inhibition of the DNAdependent bacterial RNA polymerase. ${ }^{16}$ Furthermore, rifampicin is the only potent antibiotic against Staphylococcusformed biofilms. ${ }^{17}$ Due to these excellent properties, rifampicin is an optimal antibiotic to be encapsulated into microspheres and subsequently to coat mesh implants. After surgery, these coated mesh implants can deliver rifampicin to surrounding areas in which infections are most likely to arise.

In the present study, we present the design of a local drug delivery system on the surface of a PP mesh implant. Therefore, we combined the excellent antibacterial activities of rifampicin against Gram-positive and Gram-negative bacteria with the exceptional properties of PLGA microspheres to create a mesh implant coating for the surgery of abdominal wall defects, such as hernias.

\section{Materials and methods Materials}

PLGA (Resomer RG 502 H; molecular weight [MW] = 7.000-17.000) was purchased from Evonik Industries AG (Essen, Germany), and polyvinyl alcohol (PVA; Mowiol ${ }^{\circledR}$ 18-88; MW 130.000), ethyl acetate (American Chemical Society grade), Brij ${ }^{\circledR} 30$, sodium chloride $(\mathrm{NaCl})$, and rifampicin were purchased from Sigma Aldrich (St Louis, MO, USA). Dimethyl sulfoxide (DMSO) was purchased from Merck KGaA (Darmstadt, Germany). MTT was purchased from AppliChem (Darmstadt, Germany), and PBS, Dulbecco's Modified Eagle's Medium (DMEM) high glucose (with $10 \%$ fetal bovine serum, 4,5 mmol L-glutamine, $30 \mathrm{mmol}$ [4-(2-hydroxyethyl)-1-piperazineethanesulfonic acid], and 5\% penicillin-streptomycin), and Roswell Park Memorial Institute (RPMI) without phenol red media were purchased from Gibco (Darmstadt, Germany). Schaedler Broth was purchased from BD (Franklin Lakes, NJ, USA). The light-weight PP monofilament mesh implant was provided by aimecs ${ }^{\circledR} \mathrm{GmbH}$ (Pfarrkirchen, Germany).

\section{Preparation of rifampicin-loaded microspheres}

Microspheres were prepared using the evaporation method in a laboratory scale as described previously by Reinbold et al. ${ }^{18}$ Briefly, a stable oil-in-water $(\mathrm{O} / \mathrm{W})$ emulsion was prepared to develop microspheres. Therefore, two different phases were needed: the continuous phase (CP) and the dispersed phase (DP). The $\mathrm{CP}$ contained $0.9 \% \mathrm{NaCl}$ and $1 \%$ PVA. CP was created by adding the amounts of $\mathrm{NaCl}$ and PVA to distilled water while stirring and heating. The solution had to cool down to room temperature before further use. The DP included PLGA Resomer ${ }^{\circledR}$ RG 502 H (25\%), rifampicin (1\%), and $\mathrm{Brij}^{\circledR} 30$ as an emulsifier. The two components, PLGA and rifampicin, were dissolved under continuous stirring in ethyl acetate. Brij ${ }^{\circledR} 30$ was added 
followed by slowly pipetting $(5 \mathrm{~mL} / \mathrm{min})$ the $\mathrm{DP}$ into the $\mathrm{CP}$ at 1,000 rpm at a ratio of 1:40 for emulsification. After completely injecting the DP, the stirring speed was reduced to $600 \mathrm{rpm}$ for 2 hours and then to $350 \mathrm{rpm}$ for 22 hours. After the complete evaporation of ethyl acetate, the hardening process was terminated. Subsequently, the manufactured microspheres were washed three times, separated by filtration, and air-dried. Unloaded microspheres were manufactured in the same way, except adding rifampicin to the DP.

\section{Particle size distribution}

Size and shape of the microspheres were measured in triplicates using a Mastersizer 2000 (Malvern, Worcestershire, UK) by laser diffraction according to International Organization for Standardization 13320. Therefore, microspheres were dispersed in $1 \mathrm{~L}$ distilled water, and the particle size distribution was determined by the intensity and deflection rate of a laser beam. For evaluation, Fraunhofer approximation was used. Beside particle size distribution, the parameter $\mathrm{D}(50)$ was obtained and assessed.

\section{Drug content and encapsulation efficiency of microspheres}

To determine the amount of embedded rifampicin in microspheres as well as the entrapment efficiency, $5 \mathrm{mg}$ of the rifampicin-loaded microspheres were dissolved in DMSO and the measurement was done by ultraviolet-visible (UV/ Vis) spectroscopy (BioTek EON ${ }^{\mathrm{TM}}$, Winooski, VT, USA). For this purpose, a linear calibration curve was prepared with 7 different concentrations ranging from 0.005 to $0.1 \mathrm{mg} / \mathrm{mL}$ of rifampicin dissolved in DMSO or PBS. The measurement was carried out at $\lambda=483 \mathrm{~nm}$. For this study, only $1 \%$ rifampicin was appointed. All the experiments were performed in triplicate.

$$
\begin{aligned}
& \text { Encapsulation } \\
& \text { efficiency (\%) }
\end{aligned}=\frac{\text { Actual drug loading }}{\text { Theoretical drug loading }} * 100 \%
$$

\section{Microscopy}

The manufactured microspheres were investigated using a scanning electron microscope (SEM; LEO 1450VP; Karl Zeiss Microscopy, Jena, Germany).

\section{In vitro drug release}

The in vitro drug release was measured by UV/Vis spectroscopic analysis. Therefore, $10 \mathrm{mg}$ rifampicin-loaded microspheres were filled in 50-mL tubes together with $15 \mathrm{~mL}$ PBS and incubated for 24 hours at $37^{\circ} \mathrm{C}$. The microspheres were centrifuged, and the supernatant was investigated using a photometer (BioTek EONTM). To accomplish this, $400 \mu \mathrm{L}$ of the supernatant was transferred into cuvettes and measured at $\lambda=483 \mathrm{~nm}$. The remaining supernatant in the 50-mL tubes was discarded, and the microspheres were resuspended in $15 \mathrm{~mL}$ fresh PBS and incubated for 24 hours. The amount of rifampicin released was analyzed weekly over 60 days. The total drug release was estimated as the last point of the curve.

\section{In vitro antibacterial activity}

The antibacterial characteristics of both rifampicin-loaded and unloaded microspheres were evaluated by an agar diffusion test (ADT). For this, an overnight culture of Staphylococcus aureus RN6390 was adjusted to 0.5 McFarland standard. Agar plates were bisected and seeded with $100 \mu \mathrm{L}$ of the adjusted bacteria culture. On one half, the rifampicinloaded microsphere was placed, and on the other half, an unloaded microsphere served as a control. Subsequently, the samples were incubated for 24 hours at $37^{\circ} \mathrm{C}$, and the inhibition zone was investigated.

\section{Cytotoxicity of rifampicin-loaded and unloaded microspheres on BJ fibroblasts}

The cytotoxic properties of rifampicin-loaded and unloaded microspheres were further tested. Human fibroblasts from foreskin, ie, BJ fibroblasts (Stemgent, Cambridge, MA, USA), were seeded in DMEM at a density of $1.5 \times 10^{5}$ cells per culture plates. After incubation for 24 hours at $37^{\circ} \mathrm{C}$ and $5 \% \mathrm{CO}_{2}$, the medium was discarded, cells were washed with PBS, and fresh medium was added. Rifampicin-loaded and unloaded microspheres were placed in the respective wells with their weights ranging from 0.5 to $2 \mathrm{mg} / \mathrm{mL}$ cell medium, corresponding to a mass of rifampicin varying from $12.1 \mu \mathrm{g}$ to $48.4 \mu \mathrm{g}$, and were incubated for 24 hours. After three washing steps with PBS, an MTT assay was performed to analyze the viability of the cells. Therefore, $30 \mu \mathrm{L}$ of a $0.5 \%$ MTT/PBS solution and $300 \mu \mathrm{L}$ of RPMI medium without phenol red were added into the wells and incubated for 4 hours at $37^{\circ} \mathrm{C}$ and $5 \% \mathrm{CO}_{2}$. The medium was aspirated and replaced with $300 \mu \mathrm{L}$ DMSO for 10 minutes at $37^{\circ} \mathrm{C}$ and $5 \%$ $\mathrm{CO}_{2}$. Then, $100 \mu \mathrm{L}$ was transferred to a 96-well plate and measured at $540 \mathrm{~nm}$ with the BioTek EONTM photometer. All the experiments were performed in triplicates.

\section{Implant coating}

The PP mesh implant coating process was ensued in two steps. At first, a thin primary layer of PLGA was applied. 
Therefore, a $0.5 \%$ solution of PLGA was prepared, with ethyl acetate as a solvent, and sprayed evenly on the mesh implant using an airbrush gun. The implant was dried for 3 hours at room temperature. Then, it was dusted with the prepared rifampicin-loaded microspheres, and redundant microspheres were wiped off. Remaining microspheres and the base layer were fixated at $50^{\circ} \mathrm{C}$, whereby the compounds merged together firmly. Discs of $6 \mathrm{~mm}$ in diameter were punched out and used for in vitro and in vivo investigations.

\section{In vitro antibacterial activity of the rifampicin-coated implant}

The antibacterial characteristics of the mesh implant coated with rifampicin-loaded and unloaded microspheres were also tested by ADT. For our purpose, coated mesh implants instead of single microspheres were investigated. The antibacterial properties as well as the drug release over 30 days were evaluated. Therefore, a coated mesh implant was placed in the middle of an agar plate, beforehand seeded with $S$. aureus, and incubated for 24 hours at $37^{\circ} \mathrm{C}$. Every 24 hours the inhibition zone was analyzed, and the disc was placed on a new agar plate. Rifampicin-microsphere-coated mesh implants contained $16 \mu \mathrm{g}$ of rifampicin.

\section{In vivo mouse model}

\section{Preparation of bacteria for inoculation}

$S$. aureus RN6390 was cultivated overnight in Schaedler medium; $1 \times 10^{6}$ bacteria obtained by an overnight culture were adjusted to $0.5 \mathrm{McF}$ arland standard (BioMérieux, Marcy l'Etoile, France) and centrifuged. The pellet was washed three times in PBS and resuspended for a final concentration of $1 \times 10^{6}$ bacteria $/ 50 \mu \mathrm{L}$ PBS.

\section{Implantation of mesh implants}

All the procedures were performed in accordance with institutional and federal guidelines, including the German Animal Welfare Act (TierSchG), and were approved by the Animal Care Committee (Eberhard Karls University, Tübingen, Germany) and the Regierungspräsidium Tübingen, Tübingen, Germany (approval number C6/14).

Twelve female C57BL/6J wild-type mice with an age of 6-8 weeks were used, which were purchased from the Jackson Laboratory (JAX, Bar Harbor, ME, USA). The mice were divided into three groups so that four animals were kept together in one cage. The first group received coated mesh implants with unloaded microspheres $(n=4)$, the second group was implanted with coated mesh implants with rifampicinloaded microspheres $(n=4)$, and the third group received uncoated mesh implants as a control $(n=4)$.
The mice were anesthetized with a three-component anesthesia (fentanyl, $0.05 \mathrm{mg} / \mathrm{kg}$; midazolam, (5 mg/kg; and medetomidine, $0.5 \mathrm{mg} / \mathrm{kg}$ ) via intraperitoneal injection. For pain relief after waking up, meloxicam $(1 \mathrm{mg} / \mathrm{kg})$ was injected subcutaneously. The left flank was shaved and disinfected with iodine solution (Braunoderm ${ }^{\circledR}$, B. Braun Medical AG, Sempach, Switzerland) and 70\% ethanol. Then, anesthetized mice were placed on a heating pad to prevent a drop in body temperature. Furthermore, a skin incision with a length of $8 \mathrm{~mm}$ was made using a sterile scalpel. A subcutaneous tunnel was prepared, and the mesh implant disc with a diameter of $6 \mathrm{~mm}$ was placed inside. Subsequently, the implants were covered with skin, and the incision was sutured. Then, the implants were inoculated with $50 \mu \mathrm{L}$ of the prepared $S$. aureus suspension $\left(1 \times 10^{6}\right.$ bacteria) by subcutaneous injection. The anesthesia was antagonized with a mixture of naloxone, flumazenil, and atipamezole. After 7 days, the animals were euthanized, and the implants were removed for macroscopic and histological investigations.

\section{Histological and scanning microscope analysis}

The removed implants were divided for further analyses. One half was fixed in a 4\% neutral buffered paraformaldehyde solution for 24 hours. For dehydration, the fixed implants were washed in PBS and dehydrated in a graded series of ethanol up to $100 \%$. For histochemical investigations, the samples were embedded in Technovit ${ }^{\circledR} 7200$ (Heraeus Kulzer, Wehrheim, Germany). The dehydrated implant was preinfiltrated in ethanol/Technovit ${ }^{\circledR} 7200(1: 1)$ and in pure Technovit ${ }^{\circledR} 7200$, both overnight at $4^{\circ} \mathrm{C}$ in a desiccator. Then, the samples were placed in a small dish and filled up with Technovit $^{\circledR} 7200$. Curing procedure with white light was performed in an EXAKT 520 chamber (Norderstedt, Germany) for 10 hours, followed by 10 hours with UV light.

For analysis, the polymerized blocks were cut into 100-200 $\mu \mathrm{m}$ slices with a diamond band saw (EXAKT 300/310). The slices were adhered onto a glass slide, grinded and polished with a grinding system (EXAKT 400CS), and stained with H\&E. Therefore, each slice was cleaned with a solution of acetone/ethanol (1:1), and the nuclei were stained with hematoxylin for 30 minutes followed by bluing under warm water for 15 minutes. The slices were placed in eosin dissolved in ethanol for 5 minutes, washed subsequently with water, dried on air, and investigated under a light microscope (Keyence VHX 5000; Keyence Germany GmbH, Neu-Isenburg, Germany).

The other half was fixed in $2 \%$ neutral buffered glutaraldehyde solution for 24 hours. For dehydration, the fixed implants were washed once in PBS, dehydrated in a graded 
series of ethanol up to $100 \%$, and dried with a critically point dryer (E3100 CPD; Quorum Technologies Ltd., Laughton, England). Then, dried implants were mounted on small stamps, sputtered with gold/palladium, and investigated by an SEM (LEO 1430; Karl Zeiss Microscopy).

Mice were investigated additionally with respect to their wound healing process. After euthanization, wounds were examined regarding their size and texture.

\section{Statistics}

Gaussian distribution was assumed for the MTT assay data $(n=3)$, which are depicted as means with standard errors of the mean. Hence, data were analyzed using repeatedmeasures one-way analysis of variance with Dunnet's multiple comparison test to analyze differences between groups. All the analyses were performed using the statistical software package GraphPad Prism (Version 6; GraphPad Software, La Jolla, San Diego, CA, USA). Statistical significance was defined as $p<0.05$.

\section{Results}

\section{Microsphere preparation}

Constant amounts of PLGA (25\%) and rifampicin (1\%) were used in all sets of experiments during the manufacturing process. Microspheres showed various size distributions and a spherical shape. No differences between rifampicin-loaded and unloaded microspheres were detectable (Figure 1).

\section{Particle size distribution}

The median particle size distribution was determined at $\mathrm{D}(50)$ for three individual batches (Figure 2). That means that $50 \%$ of the particles are smaller and $50 \%$ are bigger than the specified value. The average values of $\mathrm{D}(50)$ were evaluated
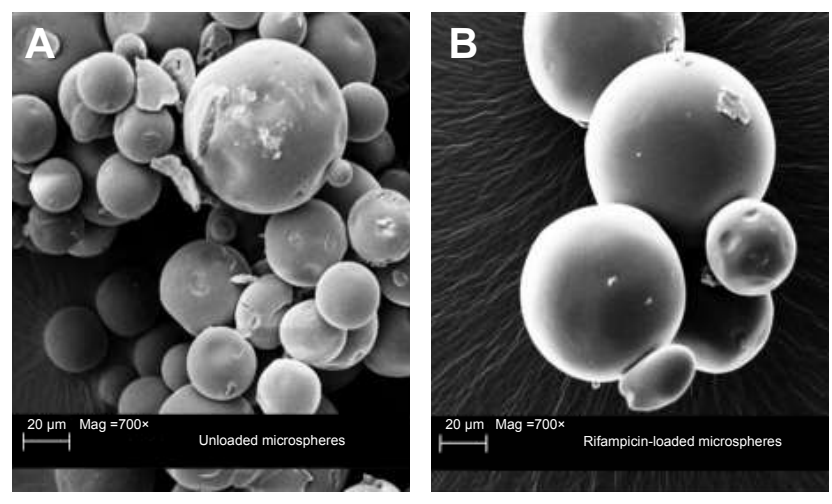

Figure I Scanning electron microscope image of the different microspheres. Notes: Scanning electron microscopic image of unloaded microspheres (A) with a magnification of $700 \times$ and rifampicin-loaded microspheres $(\mathbf{B})$ with a magnification of $700 \times$. as $67.93 \pm 3.39 \mu \mathrm{m}$ for rifampicin-loaded microspheres and $64.43 \pm 3.61 \mu \mathrm{m}$ for unloaded microspheres.

\section{Encapsulation efficiency}

The determined encapsulation efficiency using 1\% rifampicin during the preparation was $61.5 \% \pm 2.58 \%$. The drug content in $1 \mathrm{mg}$ of rifampicin-loaded microspheres was determined to be $23.71 \pm 0.53 \mu \mathrm{g}$.

\section{In vitro drug release}

The kinetic drug release of rifampicin-loaded microspheres (Figure 3) was measured over a time period of 60 days. A total drug release of $92.40 \pm 3.54 \%$ was detected by UV/Vis spectroscopy. The values of unloaded PLGA microspheres were deducted from the values of rifampicinloaded microspheres.

\section{In vitro antibacterial activity}

The antibacterial activity of rifampicin-loaded microspheres as well as mesh implants coated with rifampicin-loaded microspheres against $S$. aureus was evaluated by ADT.

No inhibition zone was detectable around the unloaded microsphere investigated by ADT (Figure 4Ai), whereas the rifampicin-loaded microsphere induced a clear inhibition zone of $14 \mathrm{~mm}$ (Figure 4Aii). The red arrow in both pictures points to a single microsphere.

In Figure 4B, the antibacterial efficiency of the coated implants over 30 days is demonstrated with an ADT test. The zone of inhibition around the implants varied from $15 \mathrm{~mm}$ to $1 \mathrm{~mm}$ over time. In contrast, the uncoated implant shows no inhibition zone (data not shown); indeed, the implant was completely covered with bacteria.

\section{Cytotoxicity of rifampicin-loaded and unloaded microspheres on BJ fibroblasts}

An MTT assay was carried out to detect potential cytotoxic effects induced by different amounts of rifampicin-loaded microspheres and unloaded microspheres on BJ fibroblasts. Figure 5 indicates no significant difference between the loaded microspheres and the control. All cells grew uniformly and did not display any uncharacteristic features; hence, cell viability was not altered by rifampicin.

\section{Wound examination}

Wounds caused by $S$. aureus infection in mice were macroscopically investigated. One mouse in the unloaded PLGA microspheres group had to be euthanized after 3 days because of a great open, weeping wound. The other 11 mice were euthanized regularly after 7 days. The wound healing 

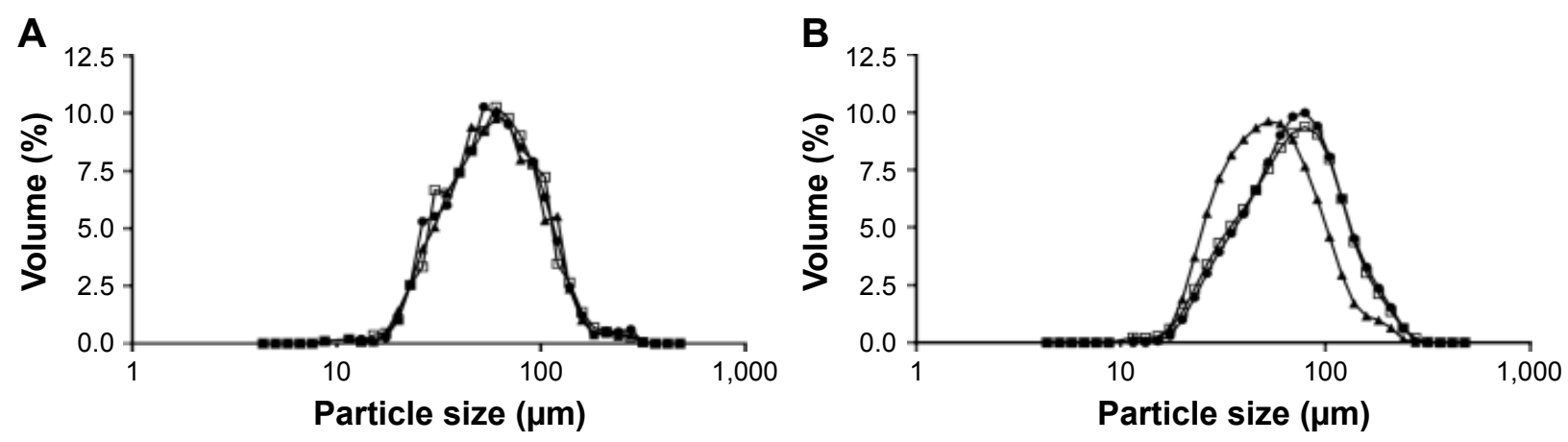

- Batch $1 \notin$ Batch $2 *$ Batch 3

Figure 2 Measurement of the particle size distribution of the different manufactured microspheres.

Notes: Particle size distribution of unloaded microspheres ( $\mathbf{A} ; \mathrm{n}=3$ batches) and rifampicin-loaded microspheres (B; $\mathrm{n}=3$ batches) measured via a Mastersizer 2000 . The unloaded microspheres have a median diameter of $64.43 \pm 3.61 \mu \mathrm{m}$ and rifampicin-loaded microspheres have a median diameter of $67.93 \pm 3.39 \mu \mathrm{m}$.

processes between the different investigation groups, which received an uncoated mesh implant (Group 1), a mesh implant coated with unloaded microspheres (Group 2), or a mesh implant coated with rifampicin-loaded microsphere (Group 3), were macroscopically observed and showed clear differences. Whereas the wounds of the mice treated with mesh implants coated with rifampicin-loaded microspheres were totally closed, the animals in the other groups showed open wounds and signs of inflammation.

\section{Histological and SEM analyses of explanted implants}

Figure 6 shows the SEM investigation of the explanted mesh implants. Figure 6A displays a good ingrowth of an uncoated mesh implant into the surrounding tissue. In one mouse of Group 1, a biofilm formation could be detected on the mesh implant surface (Figure 6B). Figure 6C and D also exhibit a great biofilm formation on the surface of an unloaded microspheres-coated mesh implant and was also found in

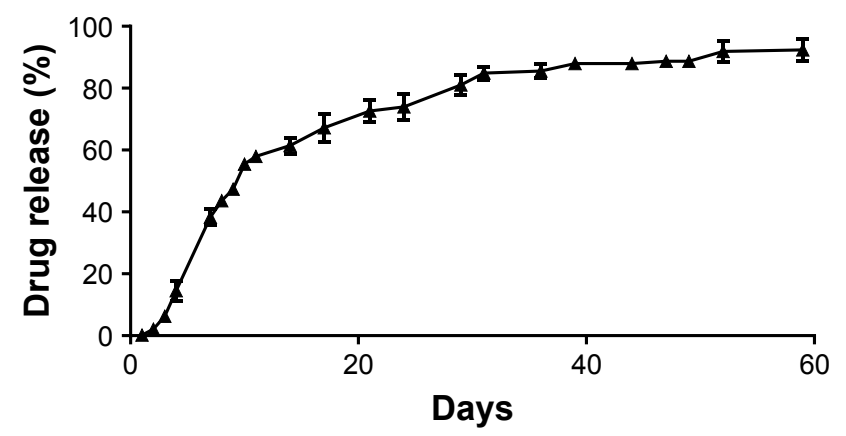

Figure 3 Investigation of the in vitro drug release from rifampicin-loaded microspheres.

Notes: In vitro kinetic drug release study from rifampicin-loaded microspheres incubated in PBS at $37^{\circ} \mathrm{C}$ over 60 days $(n=3)$. Data are given as mean with standard error of mean. one animal of Group 2. The third group showed no bacterial infection in all investigated animals (Figure 6E and F).

The pathological examination of the histological tissue sections revealed more detailed results. Overall, two of the four animals in the group with the uncoated implants revealed a strong neutrophil granulocyte infiltration of the tissue, indicating a severe acute inflammation. Furthermore, bacterial infection was also found in these animals (Figure 7A and B). In the unloaded PLGA microspheres-coated mesh implant group, bacterial infection and accompanying neutrophil granulocyte infiltration of the tissue were found in three of the four animals (Figure 7C and D). In comparison, no bacteria or neutrophil granulocyte infiltration of the tissue could be detected in the rifampicin-loaded microspheres-coated mesh implant group (Figure 7E and F).

Considering all data, ie, data from macroscopic, SEM, and histological analyses, the infection rate of the two control groups were slightly $>50 \%$, whereas no infection (four of the four animals) in the rifampicin-loaded microspheres-coated mesh implant group was detected.

\section{Discussion}

Postoperative wound infections are associated with up to $15 \%$ of all nosocomial infections. ${ }^{19}$ Accordingly, the prevention of postoperative wound infections is the first step to reduce the costs and increase the patient's compliance. With regard to postoperative wound infections, caused by mesh implants in up to $9 \%$ of cases, ${ }^{20}$ antibacterial coatings may decrease mesh-implant-associated infections, triggered by several different bacteria. ${ }^{21}$ Engelsman et al described that the main pathogens for mesh contamination are S. aureus $(90 \%)$ followed by Staphylococcus epidermidis, Escherichia coli, and Pseudomonas aeruginosa. ${ }^{22}$ In order to antagonize the 
A
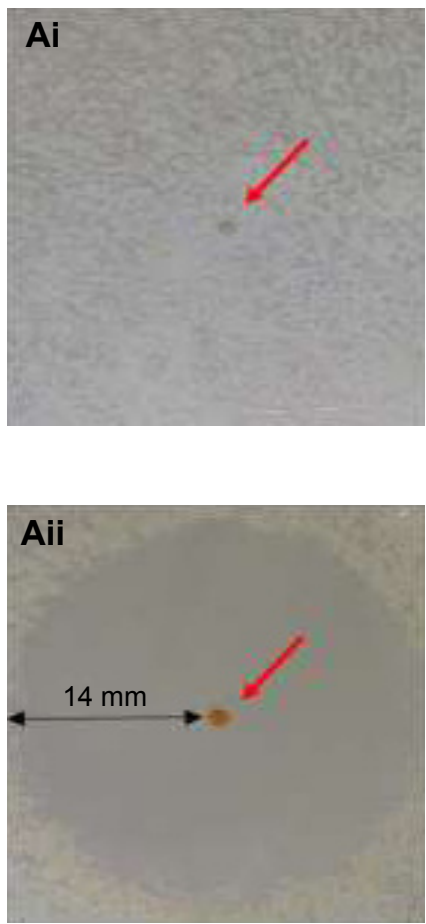

B

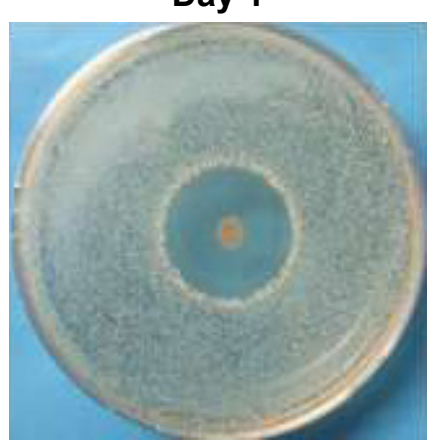

Day 20

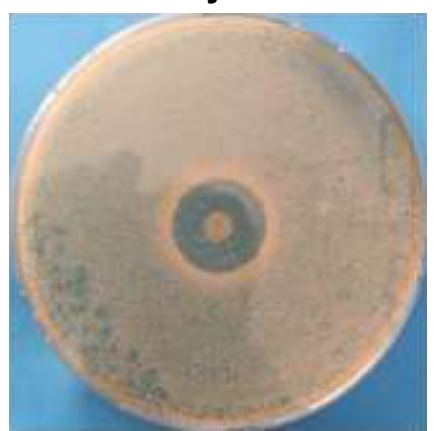

Day 10

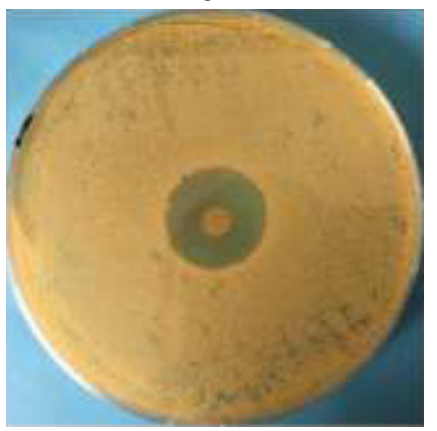

Day 30

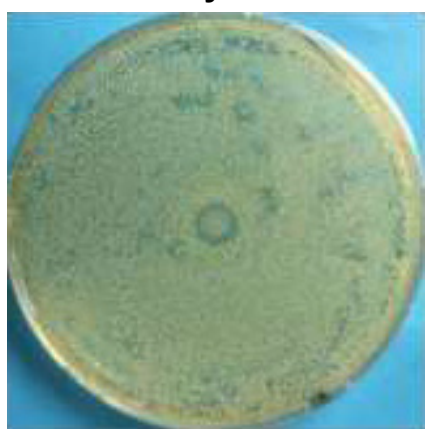

Figure 4 Agar diffusion test with unloaded and rifampicin-loaded microspheres.

Notes: Investigation of the antibacterial effect of rifampicin-loaded microsphere (Aii) in comparison with the unloaded microsphere (Ai). The inhibition zone around the rifampicin-loaded microsphere amounts to $14 \mathrm{~mm}$. Agar diffusion test with rifampicin-loaded microspheres-coated mesh implant discs (B), performed to investigate the antibacterial efficiency over 30 days. The inhibition zones vary between $15 \mathrm{~mm}$ after one day and I $\mathrm{mm}$ after 30 days.

contamination issues, systemic antibiotics are administrated, which still remains controversial, because of resistance formation. Moreover, it was shown that systemic administration does not lead to complete treatment of infection, and hence, only implant removal will alleviate symptoms. ${ }^{23}$ Furthermore, the active ingredient rifampicin is one of the

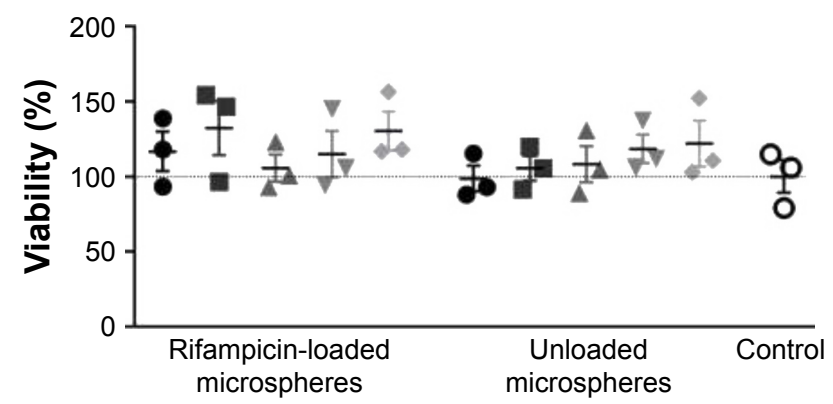

Figure 5 MTT assay of unloaded and rifampicin-loaded microspheres $(n=3)$. Notes: MTT assay was performed with BJ fibroblast after 24 hours of incubation at $37^{\circ} \mathrm{C}$ with $0.5 \mathrm{mg}, 0.75 \mathrm{mg}, 1 \mathrm{mg}, 1.5 \mathrm{mg}$, and $2.0 \mathrm{mg}$ rifampicin-loaded microspheres and unloaded microspheres per $\mathrm{mL}$ cell medium to investigate the cytotoxicity. The experiment was performed in triplicates. Data are shown as scatter plot with SEM. Abbreviation: SEM, scanning electron microscopy.

few antibiotics with substantially higher rates to eradicate staphylococcal-induced biofilms, ${ }^{24}$ but it normally has to be combined with other antibiotics to prevent the formation of bacterial resistance..$^{25}$

Therefore, the aim of the present study was to implement a novel antibacterial coating on a PP mesh implant using rifampicin-loaded PLGA microspheres. These microspheres were evaluated with respect to cytotoxicity, antibacterial activity, as well as particle size distribution. To investigate the long-term efficacy of rifampicin entrapped in the microspheres, the drug release in combination with the encapsulation efficiency was analyzed. Furthermore, the microspheres were coated on mesh implants for antibacterial testing in in vitro and in vivo models. To evaluate the efficiency, the coated mesh implant was also investigated in vitro for its long-term drug release over 60 days. Normally, release kinetics are performed in two different steps: diffusion and erosion. The diffusion mechanism correlates with loosely bound drug on the surface of the microspheres; hence, the drug can be liberated quickly, which leads to a burst release. ${ }^{26}$ Despite the solubility of rifampicin in water, no burst release was detected. The subsequent release is controlled mainly by the erosion of the microspheres. Thereby, ester bonds of the 
A

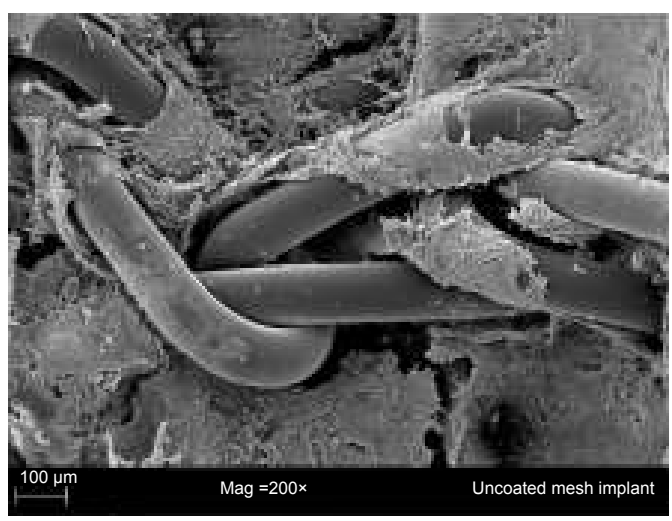

C

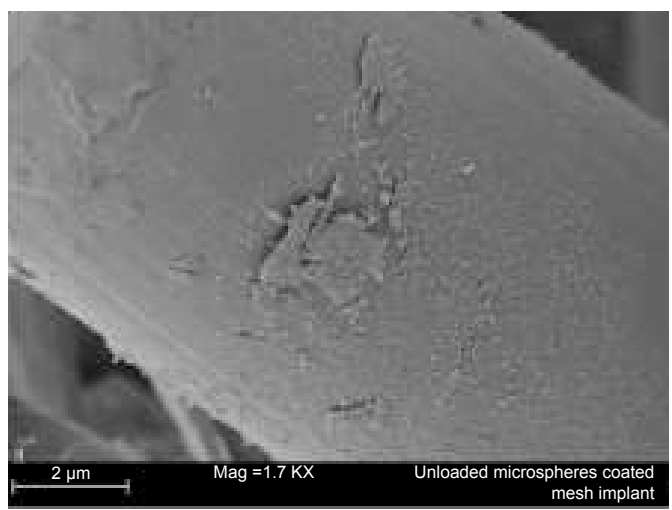

E

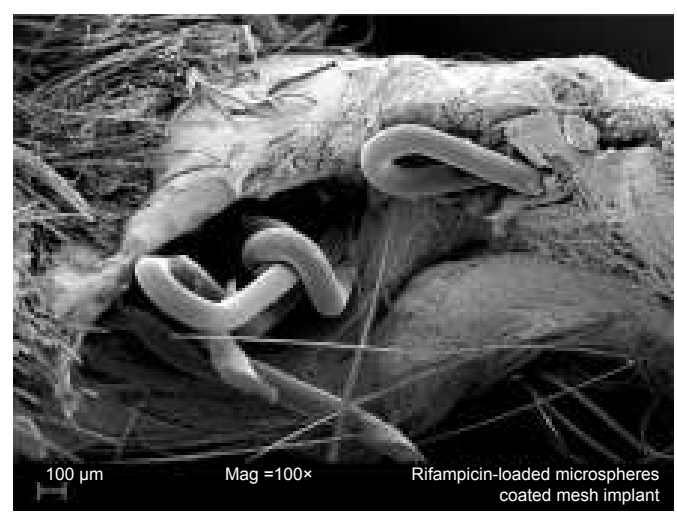

B

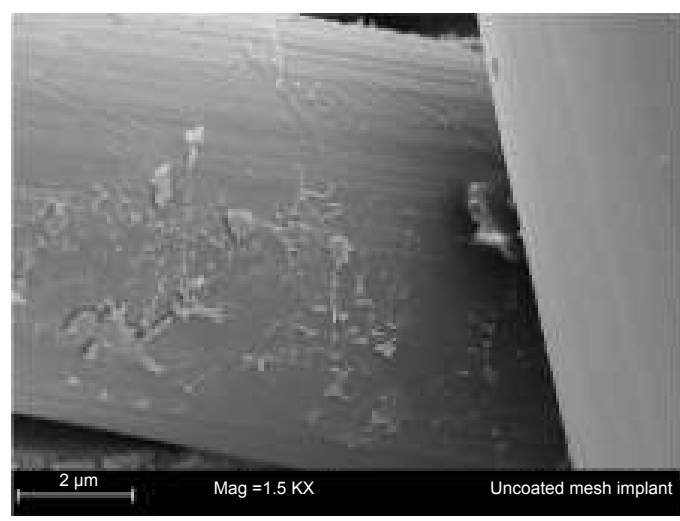

D

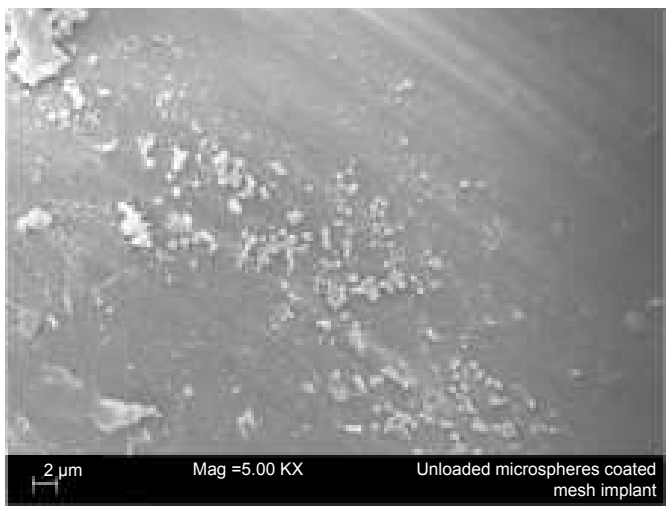

F

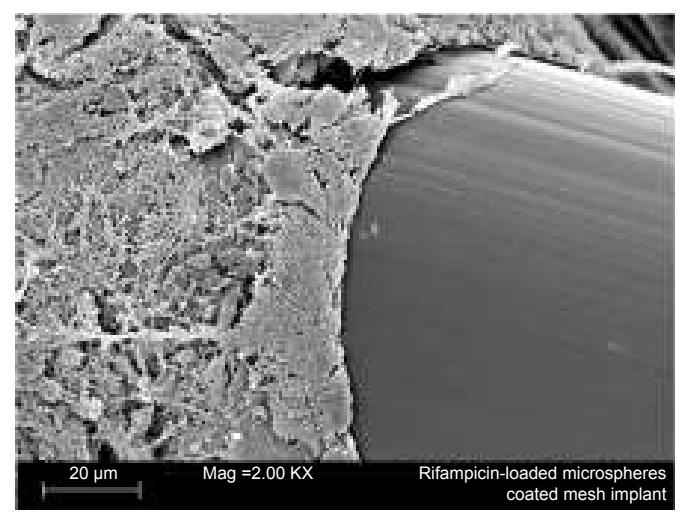

Figure 6 Scanning electron microscopy micrographs of different explanted mesh implants.

Notes: A survey of an uncoated mesh implant (A), surface with adhered bacteria of an uncoated mesh implant (B), and an unloaded microsphere coated mesh implant (C and D). No bacteria adhere on the surface of the rifampicin-loaded microsphere coated mesh implant (E and F).

polymer backbone are cleaved, and individual parts of the microspheres are fragmented due to erosion. The chemical degradation of PLGA is mediated by spontaneous hydrolysis of the ester bonds. ${ }^{26-28}$ In our analysis, prepared microspheres showed a total release of $\sim 93 \%$ after 60 days.

To get an impression of the morphology, the microspheres were analyzed due to their surface and particle size. The morphology study showed uniform particles with a smooth surface and various particle sizes. The particle distribution indicated an average median diameter of $67.93 \pm 3.39 \mu \mathrm{m}$ for rifampicin-loaded microspheres, compared with an average median diameter of $64.43 \pm 3.61 \mu \mathrm{m}$ for unloaded microspheres. The size of the microspheres as well as the entrapment efficiency can be influenced by changing several parameters during the production, eg, the rotational speed of the stirrer, the ratio of PLGA to active ingredient, the choice of solvent, and the porosity of the particles..$^{15,28}$

Moreover, as also shown in our previous study, where a natural antibacterial agent was encapsulated in PLGA microspheres, ${ }^{18}$ our method is easily adoptable to encapsulate any liposoluble drug/agent using the single emulsion $(\mathrm{O} / \mathrm{W})$ manufacturing technique.

To investigate the cytotoxicity of microspheres, an MTT assay was performed. Ignatius and $\mathrm{Claes}^{9}$ stated that the 
A

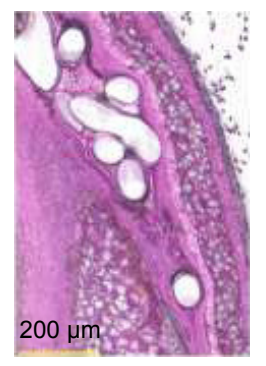

C

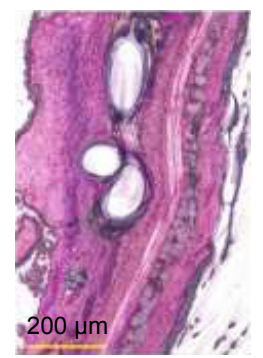

$\mathbf{E}$

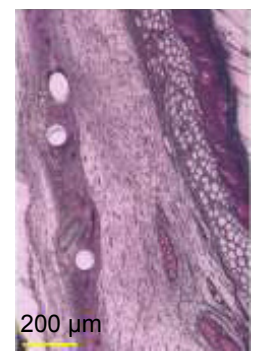

B

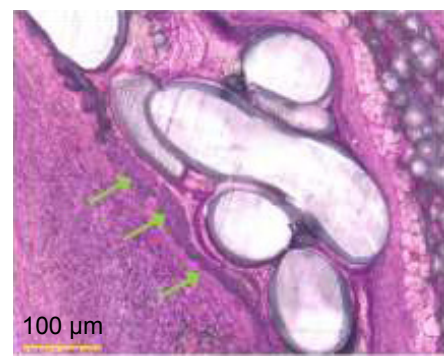

D

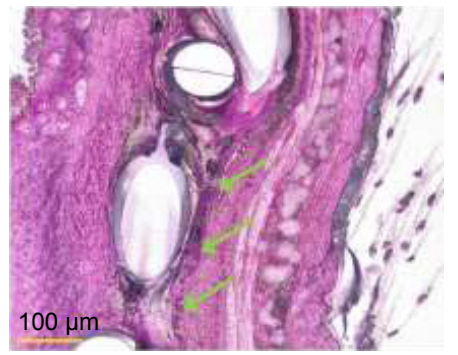

$\mathbf{F}$

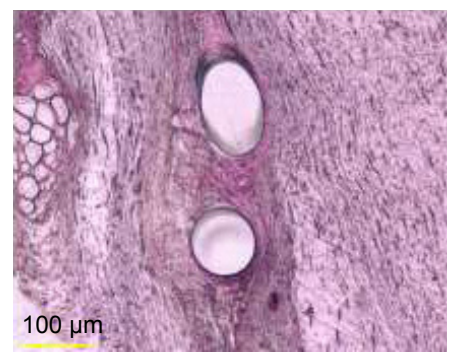

Figure 7 Histological evaluation of Technovit ${ }^{\circledR} 7200$ imbedded, explanted mesh implants.

Notes: The explanted mesh implants showed cluster of bacteria on uncoated (A, magnification 400 $\times$ ) and unloaded PLGA microspheres-coated (C, magnification 400X) mesh implants. Detailed histology shows (B, magnification I,000X): the presence of bacteria (green arrow) on an uncoated mesh implant; (D, magnification 600x): bacteria growth on the surface of an unloaded PLGA microspheres-coated mesh implant. Bacteria were found in clusters. The rifampicin-coated mesh implants (E, magnification 300×; F, magnification 600x) investigation resulted in no bacteria being found on the implant surface or the surrounding tissue.

$\mathrm{pH}$ value of cell media remains unchanged during incubation with PLGA; hence, a decrease in the $\mathrm{pH}$ value of the media was not expected. The MTT assay, which investigates the nicotinamide adenine dinucleotide phosphate-oxidasedependent cytosolic oxidases activity, showed no cytotoxic effect of different values of rifampicin-loaded or unloaded microspheres on fibroblasts. However, other adverse effects cannot be completely excluded and should be assessed in the future.

A previous study by Engelsman et $\mathrm{a}^{22}$ described different mesh implant and medical device coatings for infection prophylaxis and biofilm prevention. In the present study, the coating was achieved by means of a spray procedure with an airbrush gun. Another coating method is dip coating, which was described by Elsner et al. ${ }^{29}$ However, in our study, dip coating was not possible due to the microspheres. Consequently, the coating process was ensued in two steps, whereby first a primary layer was formed with an airbrush gun, and in the second step, the rifampicin-loaded microspheres were merged with the primary layer using a heat gun. After coating, sterilization was performed by gamma irradiation on dry ice. Stem or dry heat sterilization is an alternative technique; nevertheless, the physical and chemical properties of PLGA are altered. Conversely, chemical sterilization with ethylene oxide could influence PLGA and the active ingredient in a toxicological way. ${ }^{30}$

With regard to antibacterial efficiency, our in vitro data indicate that implants coated with rifampicin-loaded microspheres effectively inhibit $S$. aureus growth and consequently may prevent serious infections in vivo. Hence, we performed an in vivo study using a mouse model comprising three groups: 1) uncoated mesh implants, which served as control; 2) coated mesh implants with unloaded microspheres; and 3) coated mesh implants with rifampicin-loaded microspheres. The macroscopic screening showed excellent antibacterial properties of the rifampicin-loaded microsphere coating in comparison with the uncoated and the unloaded microspheres-coated mesh implants against $S$. aureus, which was confirmed by SEM investigation.

\section{Conclusion}

Mesh implants, to reinforce soft tissue like abdominal wall defects, are extensively used. To improve prophylaxis of implant-related infections and complications, new strategies need to be developed. In this study, an antibacterial coating with rifampicin embedded in PLGA microspheres was investigated. The long-term drug release over 60 days in combination with the excellent antibacterial effects, which were detectable over 30 days in vitro, showed that the coating has great potential to improve surgical site infections after the implantation of a mesh. The in vivo investigation confirmed the antibacterial effects of the mesh implants coated with rifampicin-loaded microspheres and additionally resulted in an accelerated wound healing process, which was confirmed by the SEM and histological investigations.

Overall, the rifampicin/PLGA microsphere coating on mesh implants could inhibit postoperative infections effectively over a long period without any cytotoxic effects. This could be one new strategy to prevent infections after surgical operations.

\section{Acknowledgments}

The bacterial strain (S. aureus RN6390) was a kind gift from Professor Doctor Andreas Peschel (Interfaculty Institute for Microbiology and Infection Medicine, Tübingen). The authors kindly acknowledge the excellent cooperation and 
generous support from aimecs ${ }^{\circledR} \mathrm{GmbH}$. Further, we would like to thank Helena Hinkel for technical support.

\section{Disclosure}

The authors report no conflicts of interest in this work.

\section{References}

1. Guillaume O, Garric X, Lavigne JP, Van Den Berghe H, Coudane J. Multilayer, degradable coating as a carrier for the sustained release of antibiotics: preparation and antimicrobial efficacy in vitro. $J$ Control Release. 2012;162(3):492-501.

2. Celdrán A, Esteban J, Mañas J, Granizo JJ. Wound infections due to Mycobacterium fortuitum after polypropylene mesh inguinal hernia repair. J Hosp Infect. 2007;66(4):374-377.

3. Matthews MR, Caruso DM, Tsujimura RB, Smilack JD, Pockaj BA, Malone JM. Ventral hernia synthetic mesh repair infected by Mycobacterium fortuitum. Am Surg. 1999;65(11):1035-1037.

4. Aufenacker TJ, van Geldere D, van Mesdag T, et al. The role of antibiotic prophylaxis in prevention of wound infection after Lichtenstein open mesh repair of primary inguinal hernia: a multicenter doubleblind randomized controlled trial. Ann Surg. 2004;240(6):955-961.

5. Hedrick TL, Smith PW, Gazoni LM, Sawyer RG. The appropriate use of antibiotics in surgery: a review of surgical infections. Curr Probl Surg. 2007;44(10):635-675.

6. El-Husseiny M, Patel S, MacFarlane RJ, Haddad FS. Biodegradable antibiotic delivery systems. J Bone Joint Surg Br. 2011;93(2):151-157.

7. Cancienne JM, Tyrrell Burrus M, Weiss DB, Yarboro SR. Applications of local antibiotics in orthopedic trauma. Orthop Clin North Am. 2015;46(4):495-510.

8. Klose D, Siepmann F, Willart JF, Descamps M, Siepmann J. Drug release from PLGA-based microparticles: effects of the "microparticle:bulk fluid" ratio. Int J Pharm. 2010;383(1-2):123-131.

9. Ignatius AA, Claes LE. In vitro biocompatibility of bioresorbable polymers: poly(L, DL-lactide) and poly(L-lactide-co-glycolide). Biomaterials. 1996;17(8):831-839.

10. Shive MS, Anderson JM. Biodegradation and biocompatibility of PLA and PLGA microspheres. Adv Drug Deliv Rev. 1997;28(1):5-24.

11. Hu C, Feng H, Zhu C. Preparation and characterization of rifampicinPLGA microspheres/sodium alginate in situ gel combination delivery system. Colloids Surf B Biointerfaces. 2012;95:162-169.

12. Marquette $S$, Peerboom $C$, Yates A, et al. Stability study of full-length antibody (anti-TNF alpha) loaded PLGA microspheres. Int J Pharm. 2014;470(1-2):41-50.

13. Shukla A, Avadhany SN, Fang JC, Hammond PT. Tunable vancomycin releasing surfaces for biomedical applications. Small. 2010;6(21): 2392-2404.

14. Lee SC, Oh JT, Jang MH, Chung SI. Quantitative analysis of polyvinyl alcohol on the surface of poly(D, L-lactide-co-glycolide) microparticles prepared by solvent evaporation method: effect of particle size and PVA concentration. J Control Release. 1999;59(2):123-132.
15. Vyslouzil J, Dolezel P, Kejdusova M, et al. Influence of different formulations and process parameters during the preparation of drugloaded PLGA microspheres evaluated by multivariate data analysis. Acta Pharm. 2014;64(4):403-417.

16. Davies AJ, Lewis DA. Rifampicin in non-tuberculous infections. Br Med J (Clin Res Ed). 1984;289(6436):3-4.

17. Renz N, Perka C, Trampuz A. Management periprothetischer Infektionen des Kniegelenks [Management of periprosthetic infections of the knee]. Orthopade. 2016;45(1):65-71. German.

18. Reinbold J, Hierlemann T, Hinkel H, et al. Development and in vitro characterization of poly(lactide-co-glycolide) microspheres loaded with an antibacterial natural drug for the treatment of long-term bacterial infections. Drug Des Devel Ther. 2016;10:2823-2832.

19. Watanabe A, Kohnoe S, Shimabukuro R, et al. Risk factors associated with surgical site infection in upper and lower gastrointestinal surgery. Surg Today. 2008;38(5):404-412.

20. Laurent T, Kacem I, Blanchemain N, et al. Cyclodextrin and maltodextrin finishing of a polypropylene abdominal wall implant for the prolonged delivery of ciprofloxacin. Acta Biomater. 2011;7(8):3141-3149.

21. Schmidmaier G, Lucke M, Wildemann B, Haas NP, Raschke M. Prophylaxis and treatment of implant-related infections by antibiotic-coated implants: a review. Injury. 2006;37(Suppl 2):S105-S112.

22. Engelsman AF, van der Mei HC, Ploeg RJ, Busscher HJ. The phenomenon of infection with abdominal wall reconstruction. Biomaterials. 2007;28(14):2314-2327.

23. Collage RD, Rosengart MR. Abdominal wall infections with in situ mesh. Surg Infect (Larchmt). 2010;11(3):311-318.

24. Baldoni D, Furustrand Tafin U, Aeppli S, et al. Activity of dalbavancin, alone and in combination with rifampicin, against meticillin-resistant Staphylococcus aureus in a foreign-body infection model. Int $J$ Antimicrob Agents. 2013;42(3):220-225.

25. Schwank S, Rajacic Z, Zimmerli W, Blaser J. Impact of bacterial biofilm formation on in vitro and in vivo activities of antibiotics. Antimicrob Agents Chemother. 1998;42(4):895-898.

26. Raghuvanshi RS, Singh M, Talwar GP. Biodegradable delivery system for single step immunization with tetanus toxoid. Int J Pharm. 1993; 93(1):R1-R5.

27. Doan TV, Couet W, Olivier JC. Formulation and in vitro characterization of inhalable rifampicin-loaded PLGA microspheres for sustained lung delivery. Int J Pharm. 2011;414(1-2):112-117.

28. Berkland C, Kim K, Pack DW. PLG microsphere size controls drug release rate through several competing factors. Pharm Res. 2003;20(7): $1055-1062$.

29. Elsner JJ, Shefy-Peleg A, Zilberman M. Novel biodegradable composite wound dressings with controlled release of antibiotics: microstructure, mechanical and physical properties. J Biomed Mater Res $B$ Appl Biomater. 2010;93(2):425-435.

30. Fernández-Carballido A, Puebla P, Herrero-Vanrell R, Pastoriza P. Radiosterilisation of indomethacin PLGA/PEG-derivative microspheres: protective effects of low temperature during gamma-irradiation. Int J Pharm. 2006;313(1-2):129-135.
Drug Design, Development and Therapy

\section{Publish your work in this journal}

Drug Design, Development and Therapy is an international, peerreviewed open-access journal that spans the spectrum of drug design and development through to clinical applications. Clinical outcomes, patient safety, and programs for the development and effective, safe, and sustained use of medicines are the features of the journal, which

\section{Dovepress}

has also been accepted for indexing on PubMed Central. The manuscript management system is completely online and includes a very quick and fair peer-review system, which is all easy to use. Visit http://www.dovepress.com/testimonials.php to read real quotes from published authors. 